Nephrologe 2011 · 6:209-211

DOI 10.1007/s11560-010-0428-z

Online publiziert: 20. April 2011

(c) Springer-Verlag 2011

J. Floege

Klinik für Nephrologie und klinische Immunologie, RWTH Aachen

\title{
Neue Leitlinien zur Glomerulonephritistherapie
}

ses Hefts befinden sich die Leitlinien in einem sehr fortgeschrittenen Stadium („public review“), und es ist nicht mehr mit größeren Änderungen zu rechnen. In den 6 Beiträgen dieser Ausgabe wird jeweils der aktuelle Stand der Wissenschaft im Bereich glomerulärer Erkrankungen unter besonderer Berücksichtigung der kommenden KDIGO-Empfehlungen dargestellt.

Auch in dieser KDIGO-Arbeitsgruppe hat sich wieder dramatisch gezeigt, dass die Nephrologie unter einem Mangel an soliden klinischen Studien leidet. Dieser Mangel an Therapiestudien ist in einem Bereich wie dem der Glomerulonephritiden besonders eindrucksvoll, da hier im Gegensatz z. B. zur Anämie oder Transplantation überwiegend mit generischen Immunsuppressiva behandelt wird und das Interesse der pharmazeutischen Industrie an klinischen Studien entsprechend gering ist. Dementsprechend werden die meisten KDIGO-Empfehlungen Mineralhaushalt) bisher praktisch kei-

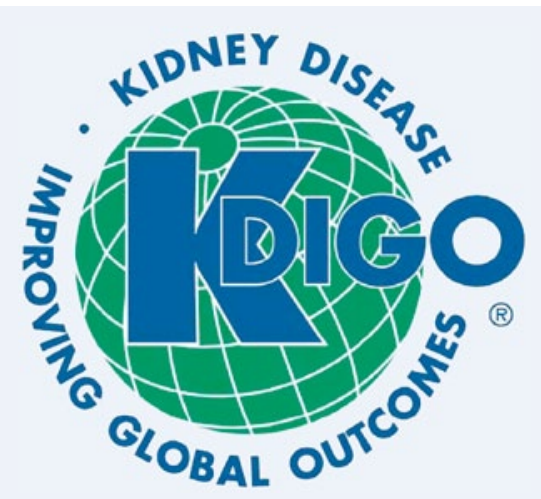

KDIGO CLINICAL PRACTICE GUIDELINE FOR GLOMERULONEPHRITIS

Work Group Co-Chairs

Abb. 1 Arbeitsgruppe zum Thema "Leitlinien in der Behandlung von Glomerulonephritiden"

\begin{tabular}{|l|l|}
\hline \multicolumn{2}{|c|}{ KDIGO CLINICAL PRACTICE GUIDELINE FOR GLOMERULONEPHRITIS } \\
Work Group Co-Chairs
\end{tabular}




\section{In eigener Sache}

nur ,... we suggest" anstelle von ,... we recommend“ (= hohes Evidenzniveau) enthalten. Fast dramatisch stellt sich die Situation bei seltenen Glomerulonephritisformen wie der membranoproliferativen Glomerulonephritis dar. Mit dieser Ausgabe von Der Nephrologe verbindet sich die Hoffnung, dass unser aller Motivation steigt, Glomerulonephritispatienten in klinische Studien wie z. B. die STOPIgAN-Studie einzuschleusen, um diesen Zustand in der Zukunft zu ändern.

Ich wünsche Ihnen viel Spaß und hoffentlich neue Einsichten bei der Lektüre.<smiles>CCCCCCCCCC</smiles>

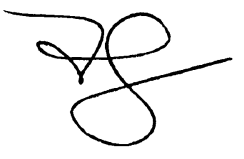

Prof. Dr. Jürgen Floege

\section{Korrespondenzadresse}

\section{Prof. Dr. J. Floege}

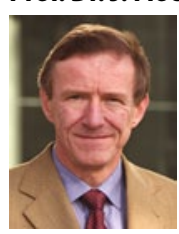

Klinik für Nephrologie und klinische Immunologie, RWTH Aachen 52057 Aachen juergen.floege@ rwth-aachen.de

\section{www.SpringerMedizin.de Das Internet der Ärzte}

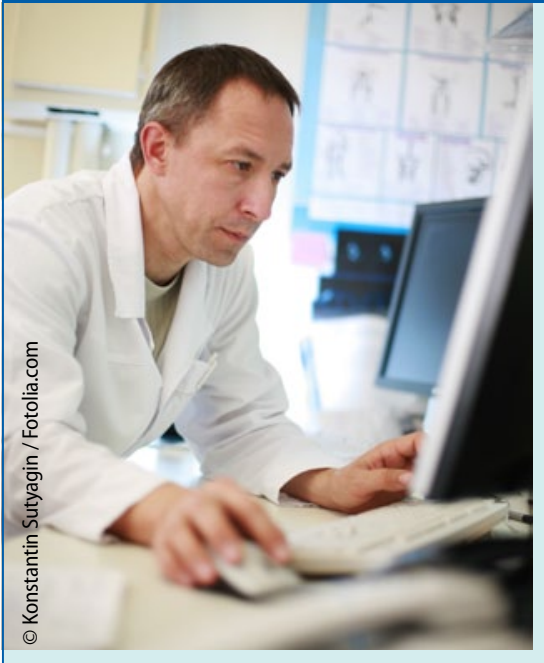

Sie möchten auf dem Laufenden bleiben? In umfassenden Zeitschriftenbibliotheken recherchieren? Sich fortbilden und CME-Punkte sammeln oder bei Experten Ihres Fachgebiets Rat suchen? Dann sind Sie auf SpringerMedizin.de, dem neuen Fachportal für Ärzte, goldrichtig. Denn dort erwartet Sie eine Fülle von nützlichen Informationen - maßgeschneidert auf Ihr Fachgebiet.
Springer Medizin nach Fachgebieten und Titeln geordnet. In dieser umfassenden Online-Bibliothek stehen für Abonnenten die Inhalte von über 70 renommierten Titeln bis in die 1990er-Jahre bereit.

Ihr personalisiertes Informationsangebot

SpringerMedizin.de ist ein Fachportal für Ärzte, daher sind alle medizinischen Inhalte passwortgeschützt. Die kostenlose Registrierung ist schnell erledigt und hat einen großen Vorteil: Sobald Sie bei der Registrierung lhr Fachgebiet angegeben haben, erhalten Sie künftig automatisch als personalisierte Startseite das entsprechende Fachportal angezeigt. Ihre Startseite können Sie nach Wunsch weiter anpassen: Die grauen Rubrikenkästen (sog. Portlets) lassen sich verschieben, umgruppieren oder auch wegklicken. Über den Knopf „Mehr Inhalte hinzufügen" können Sie weitere Rubriken auswählen und auf der Startseite anzeigen lassen. Möchten Sie auch News und Beiträge

\section{Von Anaesthesie bis Zahnmedizin - die ganze Welt der Medizin ist nur wenige Klicks entfernt. www.SpringerMedizin.de ist das Fach- portal für Ärzte in Deutschland}

Es lohnt sich, mehrmals am Tag mit SpringerMedizin.de online zu gehen. Täglich stellt Ihnen das Redaktionsteam neue interessante Themen aus Medizin und Gesundheitspolitik zusammen. Weitere News aus der Hauptstadt und Informationen, was sich in den einzelnen Bundesländern und KVen tut, stehen im Rubrikenkasten „Gesundheitspolitik" bereit. Täglich neue Meldungen zu medizinischen Themen finden Sie unter "Medizin kompakt". Kurz und bündig geben Ihnen diese Meldungen einen schnellen Überblick, was sich in der Medizin alles tut.

\section{Fortbildung und $\mathrm{CME}$}

Unter dem Navigationspunkt „Fortbildung“ stehen Ihnen interessante Übersichtsarbeiten, spannende Kasuistiken und CME-Beiträge aus den Springer-Fachzeitschriften zur Verfügung. Unter „Zeitschriften“ finden Sie das gesamte Zeitschriftenangebot von aus anderen Fachgebieten lesen - kein Problem, konfigurieren Sie Ihr Portlet ganz nach Wunsch. Sobald Sie die Konfiguration abgespeichert haben, erscheint sie beim nächsten Log-in genau so wieder. Über den orangenen Button „Guided Tour" erklären wir Ihnen alle Möglichkeiten der individuellen Anpassung in einem Film.

Diese Beispiele sind nur ein Bruchteil dessen, was SpringerMedizin.de zu bieten hat. Steuern Sie das Internet der Ärzte an und lassen Sie sich überraschen! Es lohnt sich.

\section{Dr. Sonja Kempinski}

Chefredakteurin SpringerMedizin.de 
Hier steht eine Anzeige.

黛 Springer 